\section{\\tructural analysis of macromolecular assemblies}

As proteomic and structural biology methods mature, determining highresolution structures of macromolecular

assemblies comes within reach.

A cell contains many large macromolecular assemblies, composed of multiple

\section{Controlling cells with light}

New methods using light to control cellular activity promise to illuminate hidden biology.

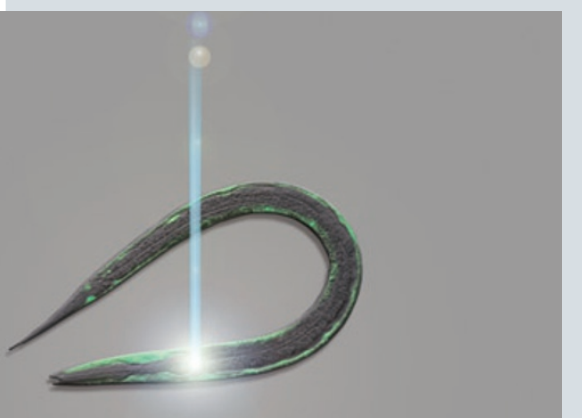

A beam of light can control the activity of a worm expressing a light-activated protein.

Light is great for looking at things, but it can also be used to alter molecules and by extension control cellular activity. In 2005 a collaboration between the laboratories of Georg Nagel and Karl Deisseroth showed that a light-activated bacterial channel called channel rhodopsin 2 (ChR2) could be used for activation of neuronal signaling. The performance and apparent ease with which the method could be implemented generated great interest among neuroscientists.

By expressing $\mathrm{ChR} 2$ in neurons and illuminating the cells with light, researchers can now depolarize neurons at will and precisely control the spiking behavior that underlies neuronal signaling.

In 2007, the same groups improved on their earlier feat by describing a new lightactivated channel that hyperpolarizes cells proteins in precise arrangements, which carry out integral biological functions. To really understand the functions of these large structures, biologists want to know what they look like, down to the atomic level. But there is not just one technology that now allows this, and researchers are beginning to take an integrated approach to tackle these large structures.

To determine what a macromolecular assembly of interest is made up of, proteomics tools, especially mass spectrom-

to inhibit neuronal signaling. They successfully used the two channels in parallel to stimulate and inhibit neuronal signaling in vivo. In a separate effort the Nagel group described a photoactivatable adenylyl cyclase, extending the technique to a new signaling pathway.

Although these papers stimulated great interest in the use of light to control cellular signaling, they were not the first such reports. Photostimulation methods have been around for years. One of the oldest, photo-uncaging, relies on the light-activated release of bioactive compounds from 'caged' compounds inactivated by a lightresponsive chemical modification. This is a powerful technique, but it relies on exogenously applied synthesized molecules and cannot take advantage of the myriad of genetic techniques for modifying cells and whole organisms.

Prior to ChR2 there were several other efforts to develop protein-based photostimulation methods. These met with some success but did not generate the groundswell of interest necessary to change the way people performed experiments.

Rapid development of ChR2-based applications by multiple groups has been instrumental in changing people's perceptions of the simplicity and utility of cellular photostimulation techniques. This high level of interest is certain to drive the development of new photo-stimulation methods and applications. It may also encourage wide use of photo-uncaging and other complementary techniques. In any case, more users will continue to adopt these methods, and the anticipated flood of reports describing biological findings has already begun.

Daniel Evanko etry-based approaches, are being used to reveal composition and stoichiometry. To obtain a portrait of the overall architecture of the assembly, cryo-electron microscopy is invaluable but has not yet yielded atomic-level structures. For this, atomic structures of the individual protein components must be modeled or determined by X-ray crystallography or NMR spectroscopy.

Fitting the pieces together like a jigsaw puzzle is perhaps the most challenging aspect. Here again, proteomics tools, including chemical cross-linking and the emerging technique of native mass spectrometry, can provide valuable information about how the components specifically interact. Rigorous computational methods are being developed to bring the data together and create structural models of macromolecular assemblies.

These structures, however, are not static entities. NMR can provide dynamic information about how the assembly functions. Cryo-electron tomography is also emerging as a noninvasive tool for analyzing large structures in situ, as they would occur in 'real life' in the cell.

In the near future, we can expect to see a growing number of examples of hybrid proteomic and structural approaches for determining high-resolution models of large macromolecular assemblies. Recently, a detailed structure of the nuclear pore complex was obtained using such an integrated approach (Nature $\mathbf{4 5 0}, 683-$ 694; 2007 and Nature 450, 695-701; 2007), which should surely serve as a model for success.

Allison Doerr

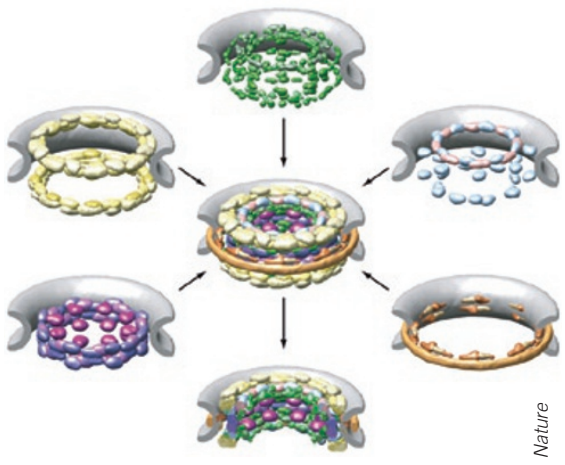

An integrated structural model for the nuclear pore complex. 